\title{
Influence of cryotherapy on balance and joint position sense in healthy subjects: randomized clinical trial
}

\author{
Caio Alano de Almeida Lins', Liane de Brito Macedo1, Renata Augusta Gomes Silveira', Daniel Tezoni Borges', \\ Jamilson Simões Brasileiro
}

\begin{abstract}
Background: Cryotherapy has been widely used in clinical practice, particularly for the treatment of acute injuries to soft tissues and various joints. However, since decrease in temperature can results in reduced nerve conduction velocity and proprioception, it can therefore be assumed that cryotherapy, applied before exercise, can result in a greater predisposition of the joints to lesion. Thus, the objective of this study was to evaluate the influence of cryotherapy on balance and knee joint position sense (JPS). Methods: We conducted a randomized single-blinded clinical trial, with thirty volunteers of both genders (age: $23.3 \pm 2.5$ years; $\mathrm{BMl}: 22.2 \pm 2.1 \mathrm{Kg} / \mathrm{m}^{2}$ ), who were randomly distributed into two groups with 15 subjects in each: control group - 20 minutes at rest; and experimental group - application of cryotherapy on the knee of the dominant lower limb, for 20 minutes. All subjects were submitted to the assessment of the balance, by means of computerized baropodometry and the JPS of the knee of the dominant lower limb (DLL) using an electrogoniometer, both open kinetic chain (OKC) and closed kinetic chain (CKC), before and after the interventions. Statistical analysis was performed using SPSS 20.0 software. Kolmogorov-Smirnov test was used to check the data normality and two-way ANOVA to verify intra and inter-group differences. The study was approved by the local Research and Ethics Committee (Approval no. 099/10). Results: There was a difference on the JPS assessed in OKC in the experimental group $(p=0.03)$. There was no alteration in the balance and the JPS with CKC, in none of the groups tested $(p>0.05)$. Conclusion: Cryotherapy when applied on knee did not affect balance or the JPS with CKC of the knee, although alteration was observed in OKC. We therefore conclude that application of cryotherapy before exercises do not posses increased risk of myoarticular injuries, since the most of these activities is performed in CKC.
\end{abstract}

Keywords: Proprioception; Kinesthesis; Mechanoreceptors.

Corresponding Author: Prof. Dr. Jamilson Simões Brasileiro. Av. Senador Salgado Filho, 3000. Campus Universitário, Lagoa Nova, Natal (RN), Zip code 59.078-970, Brazil. Phone: (084)33422008. E-mail: brasileiro@ufrnet.br

${ }^{1}$ Neuromuscular Performance Analysis Laboratory (LAPERN), Department of Physiotherapy, Universidade Federal do Rio Grande do Norte (UFRN), Natal (RN), Brazil.

Financial support: None.

Submission date 29 April 2015; Acceptance date 1 August 2015; Online publication date 10 August 2015 


\section{INTRODUCTION}

Cryotherapy has been commonly used to reduce skin ${ }^{(1)}$ and muscle ${ }^{(2)}$ temperature, to control secondary lesion due to hypoxia and edema ${ }^{(3)}$ and for pain relief. ${ }^{(4,5)}$ The cryotherapy protocols, which include application of ice and immersion in iced water, are used by coaches and physiotherapists, despite the lack of conclusive studies on the potential risks that the athletes and/or patients may face after its use. ${ }^{(6)}$

The effect of cryotherapy on proprioception, which is a component of the somatosensory system, is still poorly understood. (7) Proprioceptive acuity is defined as the ability of an individual to determine the joint position sense, motion and strength of the limbs. ${ }^{(8)}$ Consequently, the proprioceptive acuity is an essential component for injury prevention and rehabilitation, but is often ignored, with serious consequences, because the proprioceptive deficit can be responsible for many acute joint lesions. ${ }^{(9,10)}$

Proprioception is the result of afferent generated from integration of neural impulses that originate from various mechanoreceptors to the central nervous system (CNS). These receptors are located in joint capsules, ligaments, muscles, tendons and skin and are sensitive to stimuli, such as pain, pressure, touch and movement. Therefore, their role is critical to the performance of the individual in sport and daily activities. ${ }^{(11,12)}$

A number of techniques used in examining proprioceptive acuity have been described in the literature, among which is the joint position sense (JPS). Assessment of the JPS of an individual determines, in particular, its ability to realize a target angle or position of the articulation, and may be conducted in an active or passive form and by positioning in an open kinetic chain (OKC) or closed kinetic chain (CKC). ${ }^{(12,13)}$ The ability to position a joint consciously, is a highly specialized proprioceptive function and is a measure of great clinical importance, which involves both the motion control and stability. ${ }^{(14)}$

The proprioceptive information is also an important element in static and dynamic balance. This balance is defined as the capacity to maintain the center of gravity about its base of support, and depends on an adequate operation of postural control system. This complex system of feedback is based on the integration of visual afferents, vestibular and somatosensory systems, with the neuromuscular efferent. ${ }^{(15)}$ It should reach the CNS accurately in order to promote good muscle control. ${ }^{(16)}$

Therefore proprioception is a decisive element in the recovery of an individual after an injury. Beyond the proprioceptive trainings and balance, cryotherapy has been widely used in clinical practice, particularly in the treatment of acute injuries of soft tissues and various joints. ${ }^{(17,18,19)}$ However, the temperature decrease causes reduction in nerve conduction velocity (NCV) ${ }^{(20)}$ and the proprioception. ${ }^{(21)}$ Thus, it can be assumed that this therapeutic modality, if applied before exercise, can result in an inappropriate peripheral feedback for both the JPS, as well as for the body balance, which can change the biomechanical properties of the joint, resulting in a greater lesion predisposition of the joint when the exercise is resumed. ${ }^{(18)}$

Considering the above, this study aimed to analyze the immediate effects of cryotherapy application on balance and JPS of the knee in OKC and CKC exercises in healthy subjects.

\section{METHODS}

The present study is a randomized, single-blinded clinical trial. The first researcher performed the initial and final assessments of all volunteers, while the second researcher was responsible for the implementation of the protocol and a third researcher performed the statistical analysis. The study was approved by the local Research and Ethics Committee (Approval no. 099/10) and was carried out in the Neuromuscular Performance Analysis Laboratory (LAPERN) of the Department of Physiotherapy at the Universidade Federal do Rio Grande do Norte (UFRN).

Thirty healthy volunteers, of both genders, were selected for this study with an average age of $23.3 \pm 2.5$ years and average body mass index (BMI) of $22.2 \pm 2.1 \mathrm{Kg} / \mathrm{m}^{2}$. The following inclusion criteria were considered: aged between 18 and 28 , recreationally physically active ${ }^{(22)}$, integrity of the knee joint of the dominant lower limb, absence of musculoskeletal injury history of the lower limbs in the last six months, no history of lower limb surgery, no neurological deficits, no uncorrected visual and/or hearing deficits, and no allergy to cold. Volunteers who reported pain during the procedures for data collection or those that fail to implement the assessment procedures in the correct manner were excluded from the study.

Participants were recruited among students from a local university, who were informed about the study objectives and gave their informed consent in accordance with the resolution 466/12 of the Brazilian National Health Council and the declaration of Helsinki for research involving human beings.

\section{Procedures}

An initial assessment was performed evaluating one-footed static balance by means of computerized baropodometry, and the JPS of the knee in OKC and CKC. After this assessment, the volunteers were randomly distributed, using an online tool (www.randomization.com), into two groups with 15 participants each. The groups were color-coded by the second researcher, and the color corresponding to each group was revealed only after completion of the statistical analysis. The control group ( 7 men and 8 women), who went through the initial assessment, rested for 20 minutes, by lying in a supine position with knees extended, to carry out thereafter the final evaluation. 
The volunteers in the experimental group ( 7 men and 8 women) were subjected to 20 minutes of cryotherapy. The ice was applied on the knee joint of the participants by means of two ice packs of about three kilograms each. The ice packs were properly positioned, one on the anterior region and the other on the popliteal region, fastened with the aid of a non-elastic tape for proper clamping. Immediately after the intervention, the volunteers underwent a second assessment similar to the initial one, differing only in not repeating the training to determine JPS. The same procedure was applied to the control group.

\section{Assessment of JPS}

Procedure for assessment of the JPS began with fixing of the electrogoniometer (EMG System ${ }^{\circledR}$, Brazil) on the dominant lower limb (DLL), which is done as follows: 1) The volunteer remained standing and the goniometer axis was positioned on the knee joint at the level of the lateral epicondyle of the femur. Then, the fixed arm was positioned parallel to the femur toward the greater trochanter and the movable arm parallel to the fibula, toward the lateral malleolus. The goniometer was fixed with bandages and two velcro straps.

Aiming to eliminate visual afferences, the participants were blindfolded to carry out JPS assessment. JPS was first assessed in OKC. The volunteers were asked to sit on a chair and remained with the hip and the knee flexed to 90․ They were then asked to fully extend the knee. From this position (approximately 180ㅇ), they were asked to flex it slowly up to the angle of 135‥ After achieving this angulation, they were requested to remain in the position for 5 seconds and then were asked to relax the limb and remain at rest for 10 seconds. This training was performed three times and in the fourth maneuver, volunteers were asked to extend the lower limb tested to the angulation which was trained previously.

The assessment of the JPS at CKC was performed with the volunteers standing, blindfolded, in a one-footed support on the DLL and supported on the contralateral hand to avoid imbalance. The volunteers were requested to flex the knee until reaching an angle of 145ㅇ. The procedure was performed in the same manner, while maintaining the position for 5 seconds, and then remaining at rest for 10 seconds. In the fourth set, volunteers were asked to flex the knee until the trained amplitude.

The variable analyzed was the absolute error, given by the difference between the target-angle and the angle reached by the volunteer, in degrees, without considering directional tendencies of over or underestimation of target-angle.

\section{Assessment of one-footed static balance}

A $40 \mathrm{~cm} \times 40 \mathrm{~cm}$ computerized baropodometer (Eclipse 3000 , Guy-Capron ${ }^{\circledR}$ SA, França), was used to collect one-footed static balance data. Subjects were standing on the platform of the baropodometer with support on the DLL and knee flexed at 20 - as measured by an electrogoniometer. Individuals were instructed to keep their head in the neutral position, staring at a fixed point, spine erect, with upper limbs supported on the hip. The non-dominant lower limb remained with the hip at 0 ㅇ and knee flexed at 90 (figure 1). Data acquisition time was $10 \mathrm{~s}$, using a frequency of $20 \mathrm{~Hz}$. Subjects were assessed twice, with the best result considered for analysis ${ }^{(23)}$. A 1-min rest period was allowed between each test and the variables analyzed were displacement amplitude and displacement velocity of the pressure center in the antero-posterior $(A / P)$ and latero-lateral (L/L) directions.

\section{Statistical Analysis}

Statistical analysis was performed using SPSS 20.0 software. We first carried out Kolmogorov-Smirnov (K-S) test to verify data normality and we observed that all the variables presented normal distribution.

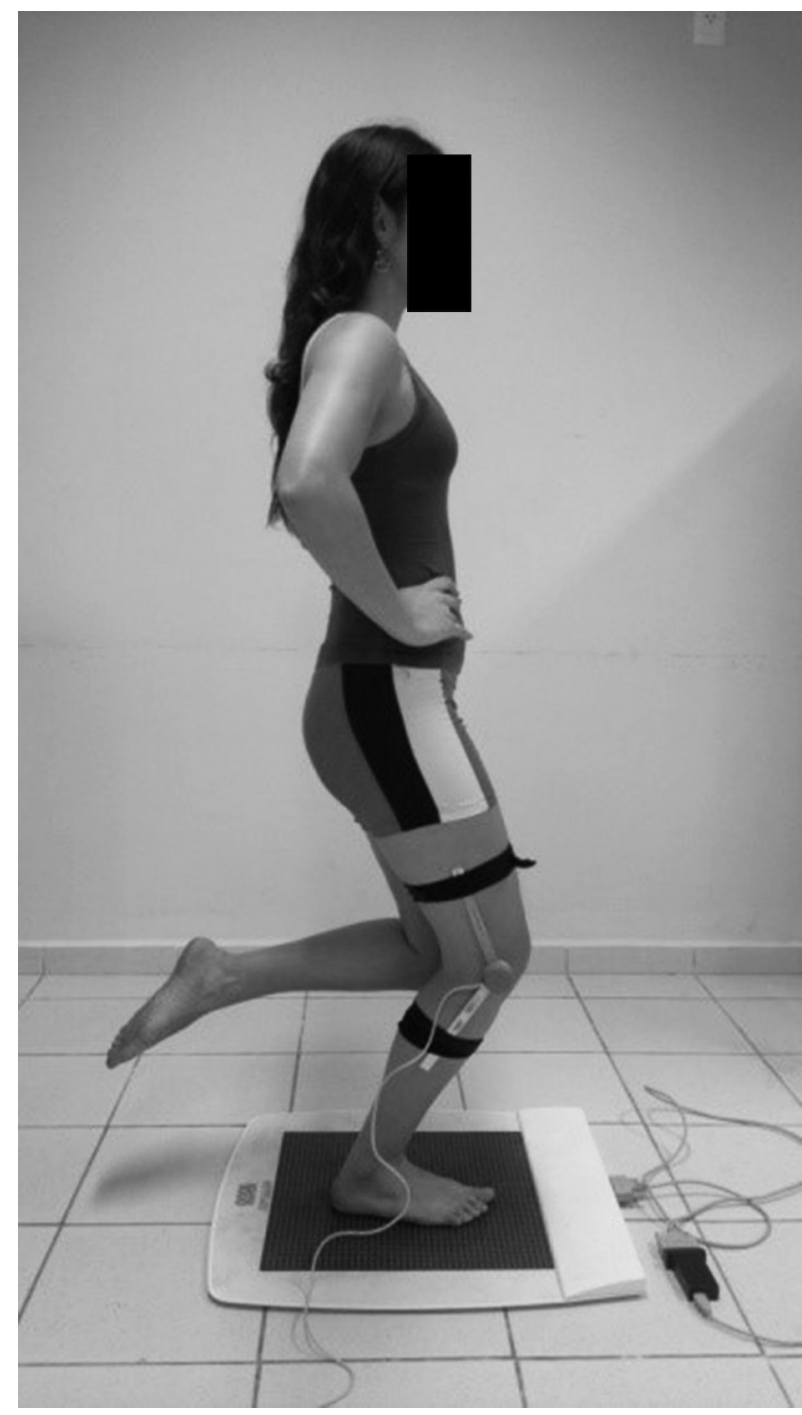

Figure 1. Volunteers position for testing the one-footed static balance. 
For inferential statistical analysis of variance, we used two-way ANOVA to check intra and inter-group differences before and after the interventions. In all the statistical analysis a significance level of $5 \%$ and a confidence interval of $95 \%$ (Cl 95\%) were assigned.

\section{RESULTS}

In relation to the one-footed static balance, we observed no significant difference between the initial and final assessments for amplitude of displacement, A/P and L/L velocity in the two groups studied (table 1). No significant difference was observed among the groups.

As far as the JPS assessed in OKC, it can be observed in Table 2 that there was a significant difference from the experimental group, when the values of the initial assessment were compared with the final evaluation. However, in the control group there was no significant difference when the initial assessment was compared with the final. In relation to the JPS assessed in CKC, we observed no significant difference between the initial and final evaluations, in any of the groups studied (table 2). No significant difference was also observed among the groups.

\section{DISCUSSION}

The finding in our study, which indicates significant changes only in JPS in OKC after cryotherapy application is similar to those found by Oliveira et $\mathrm{al}^{(21)}$, Surenkok et $\mathrm{al}^{(24)}$ and Uchio et al. ${ }^{(18)}$. They reported that the use of ice caused a reduction in NCV, delaying or even blocking the proprioception of articular capsule and the links to the higher centers of motor control. This reduction in the NCV is attributed to decrease in temperature that can alter the exchange of $\mathrm{Ca}^{2+}$,
$\mathrm{Na}^{+}$and $\mathrm{K}^{+}$ions in neural cells, resulting in delay in the generation of action potential. ${ }^{(25)}$

Proske et al. ${ }^{(26)}$ state in their review that muscle contraction increases the proprioceptive acuity in relation to passive motion. In their study Surenkok et al ${ }^{(24)}$ assessed JPS in OKC in a passive way, differing from the present study that evaluated the same through active movement of the examined limb. In both cases, cryotherapy led to reduction in the JPS when compared with the assessments made before cooling. Thus, we can reasonably argue that the participation of quadriceps muscle afference in active test was unable to supply the proprioception deficit generated by a reduction in local temperature and consequent lower NCV.

When compared with activities in OKC, some studies have demonstrated the superiority of activities in CKC in acuity of measures of JPS. ${ }^{(27)}$ This superiority may be attributed to a greater proprioception caused by increased tissue deformation, joint reaction forces (compression) and increased muscle activation (co-contraction). In addition, there is the input from the other muscles and joints involved in the movement, instead of the OKC, where the afferent information comes only from an articulation and surrounding muscles. ${ }^{(27)}$

Our study compared the changes that can occur to JPS in OKC and CKC after cryotherapy. The results showed that there were no changes in JPS assessed in CKC, even after application of cryotherapy on knee joint. This demonstrates that the proprioceptive afference from other joints (ankle and hip, for example) and muscles involved in the activity overlapped a possible reduction of joint proprioception of the knee, keeping the JPS unchanged.

The other proprioceptive aspect observed in this study was the one-footed static balance, where no significant alterations

Table 1. Displacement amplitude and velocity antero-posterior and latero-lateral of the center of pressure.

\begin{tabular}{|c|c|c|c|c|c|c|}
\hline \multirow{3}{*}{ Variables $(n=15)$} & \multicolumn{2}{|c|}{ CONTROL } & \multirow{3}{*}{$\mathbf{p}$} & \multicolumn{2}{|c|}{ EXPERIMENTAL } & \multirow{3}{*}{$\mathbf{p}$} \\
\hline & \multicolumn{2}{|c|}{ Mean \pm SD } & & \multicolumn{2}{|c|}{ Mean \pm SD } & \\
\hline & PRE & POST & & PRE & POST & \\
\hline Displacement amplitude (mm) & $62 \pm 29$ & $71 \pm 30$ & 0.86 & $65 \pm 40$ & $63 \pm 29$ & 0.86 \\
\hline Velocity A/P (mm/s) & $12 \pm 2.5$ & $12.1 \pm 3.0$ & 0.93 & $12 \pm 2.3$ & $11.5 \pm 2.3$ & 0.90 \\
\hline Velocity L/L (mm/s) & $5.5 \pm 0.5$ & $5.6 \pm 0.7$ & 0.40 & $6.0 \pm 1.9$ & $5.6 \pm 0.6$ & 0.47 \\
\hline
\end{tabular}

A/P: antero-posterior; L/L: latero-lateral. SD: Standart Deviation

Table 2. Joint position sense in open kinetic chain and closed kinetic chain.

\begin{tabular}{|c|c|c|c|c|c|c|}
\hline \multirow{3}{*}{ Variables $(n=15)$} & \multicolumn{2}{|c|}{ CONTROL } & \multirow{3}{*}{$\mathbf{P}$} & \multicolumn{2}{|c|}{ EXPERIMENTAL } & \multirow{3}{*}{$\mathbf{P}$} \\
\hline & \multicolumn{2}{|c|}{ Mean \pm SD } & & \multicolumn{2}{|c|}{ Mean \pm SD } & \\
\hline & PRE & POST & & PRE & POST & \\
\hline Absolute error in OKC (degrees) & $4 \pm 2,4$ & $4.4 \pm 3$ & 0.86 & $2.1 \pm 1.1$ & $5.2 \pm 2.1$ & $0.03^{*}$ \\
\hline Absolute error in CKC (degrees) & $2.3 \pm 1.5$ & $3.2 \pm 3.0$ & 0.37 & $3.3 \pm 3.0$ & $4.5 \pm 3.0$ & 0.17 \\
\hline
\end{tabular}

OKC: open kinetic chain; CKC: closed kinetic chain. * Significant difference when comparing the JPS values in OKC pre and post intervention, for the experimental group. 
were observed in any of the groups assessed. These results differ from those of Surenkok et al ${ }^{(24)}$, who observed changes in balance after the application of cryotherapy. They attributed their results to the fact that the cryotherapy affects the sensory afference of peripheral receptors, thus altering the balance. In fact Miniello et al $^{(28)}$ found no changes in dynamic stability of ankle after immersion of the leg in iced water, even having observed changes in muscle activity of the peroneus longus and tibialis anterior.

We suggest that, being the balance assessment an activity in CKC, the proprioceptive deficit of receptors, possibly altered by reducing the temperature was compensated for by afferences from other joints, which are optimized for this type of kinetic chain. Therefore, we did not observe changes in the one-footed static balance after application of cryotherapy.

\section{CONCLUSION}

The results obtained in this study suggest that the application of cryotherapy alter the JPS assessed in OKC, but does not influence the one-footed static balance and the knee JPS evaluated in CKC. In this manner, it is suggested that the use of cryotherapy, preceding the proprioceptive training, a common practice in physiotherapy, is not contraindicated, since this type of training is usually performed with predominance of activities in closed kinetic chain.

Finally, it is important to emphasize that the results of this study should be limited to healthy subjects engaging in recreational physical activity. Therefore, it is suggested that further studies should be performed in individuals with previous proprioceptive deficits.

\section{ACKNOWLEDGEMENTS}

For the financial assistance granted for this study, we are grateful to Conselho Nacional de Desenvolvimento Científico e Tecnológico (CNPq) and Coordenação de Aperfeiçoamento de Pessoal de Nível Superior (CAPES).

\section{AUTHORS CONTRIBUTION}

Creating the idea that originated the work and elaborate hypotheses: CAAL, LBM, RAGS, DTB, JSB. Guiding or coordinate the work: JSB. Write the manuscript: CAAL, LBM, DTB, JSB. Collect data: CAAL, RAGS. Analyze the results statistically: CAAL, LBM, JSB. Guide the writing of the manuscript: CAAL, LBM, DTB, JSB. Review the literature: CAAL, LBM, RAGS, DTB.

\section{COMPETING INTERESTS}

The authors declare no conflicts of interest.

\section{REFERENCES}

1. Janwantanakul P. The effect of quantity of ice and size of contact area on ice pack/ skin interface temperature. Physiother. 2009;95:120-5.

2. Rupp KA, Herman DC, Hertel J, Saliba SA. Intramuscular temperature changes during and after 2 different cryotherapy interventions in healthy individuals. J Orthop Sports Phys Ther. 2012;42(8):731-7.
3. Ferreira-Junior JB, Bottaro M, Loenneke JP, Vieira A, Vieira CA, Bemben MG. Could whole-body cryotherapy (below $-100^{\circ} \mathrm{C}$ ) improve muscle recovery from muscle damage? Front. Physiol. 2014;5: 247.

4. Fonda B, Sarabon N. Effects of whole-body cryotherapy on recovery after ham- string damaging exercise: a crossover study. Scand. J. Med. Sci. Sports. 2013;23: 270-8.

5. Pastre CM, Bastos FN, Netto Junior J, Vanderlei LCM, Hoshi RA. Métodos de recuperação pós-exercício: uma revisão sistemática. Rev Bras Med Esporte. 2009;15(2):138-144.

6. Vaile J, Halson S, Gill N, Dawson B. Effect of hydrotherapy on the signs and symptoms of delayed onset muscle soreness. Eur J Appl Physiol. 2008;102(4):447-455.

7. Costello JT, Donnelly AE. Cryotherapy and joint position sense in healthy participants: A systematic review. J Athl Train. 2010;45(3):306-316.

8. Muaidi QI, Nicholson LL, Refshauge KM. Proprioceptive acuity in active rotation movements in healthy knees. Arch Phys Med Rehabil. 2007;89(2):371-6.

9. Bleakley $C$, McDonough S, MacAuley D. The use of ice in the treatment of acute soft-tissue injury: a systematic review of randomized controlled trials. Am J Sports Med. 2004;32(1):251-261.

10. Zazulak BT, Hewett TE, Reeves NP, Goldberg B, Cholewicki J. The effects of core proprioception on knee injury: a prospective biomechanicalepidemiological study. Am J Sports Med. 2007;35(3):368-373.

11. Wassinger CA, Myers JB, Gatti JM, Conley KM, Lephart SM. Proprioception and throwing accuracy in the dominant shoulder after cryotherapy. J Athl Train. 2007;42(1):84-9.

12. Ribeiro F, Mota J, Oliveira J. Effect of exercise-induced fatigue on position sense of the knee in the elderly. Eur J Appl Physiol. 2007;99:379-385.

13. Dover G, Powers ME. Reliability of joint position sense and forcereproduction measures during internal and external rotation of the shoulder. J Athl Train. 2003;38(4):304-310.

14. Bennell K, Wee E, Crossley K, Stillman B, Hodges P. Effects of experimentally induced anterior knee pain on knee joint position sense in healthy individuals. J Orthop Res. 2005;23(1):46-53.

15. Hansen MS, Dieckmann B, Jensen K, Jakobsen BW. The reliability of balance tests performedon the kinesthetic ability trainer (KAT 2000). Knee Surg Sports Traumatol, Arthrosc. 2000;8:180-5.

16. Janwantanakul P, Magarey ME, Jones MA, Dansie BR. Variation in shoulder position sense at mid and extreme range of motion. Arch Phys Med Rehabil. 2001;82:840-4.

17. Bishop PA, Jones $E$, Woods AK. Recovery from training: a brief review. J. Strength Cond. Res. 2008;22:1015-1024.

18. Uchio Y, Ochi M, Fujihara A, Adachi N, Iwasa J, Sakai Y. Cryotherapy influences joint laxity and position sense of the healthy knee joint. Arch Phys Med Rehabil. 2003;84:131-5.

19. Algafly AA, George KP. The effect of cryotherapy on nerve conduction velocity, pain threshold and pain tolerance. J Sports Med. 2007;41:365-9.

20. Herrera E, Sandoval MC, Camargo DM, Salvini TF. Motor and sensory nerve conduction are affected differently by ice pack, ice massage, and cold water immersion. Phys Ther. 2010;90:581-591.

21. Oliveira R, Ribeiro F, Oliveira J. Cryotherapy impairs knee joint position sense. Int J Sports Med. 2010;31(3):198-201.

22. Pincivero DM, Gandaio GB, Ito, Y. Gender-specific knee extensor torque, flexor torque, and muscle fatigue responses during maximal effort contractions. Eur J Appl Physiol. 2003;89:134-141.

23. Ageberg E, Roberts $D$, Holmström E, Fridén T. Balance in Single-Limb Stance in Patients With Anterior Cruciate Ligament Injury: Relation to Knee Laxity, Proprioception Muscle Strength, and Subjective Function. Am J Sports Med. 2005;33:1528-1535.

24. Surenkok O, Aytarb A, Tüzün EH, Akman MN. Cryotherapy impairs knee joint position sense and balance. Isokinet and Exerc Sci. 2008;16:69-73.

25. Reid G, Babes A, Pluteanu F. A cold and menthol-activated current in rate dorsal root ganglion neurones: properties and role in cold transduction. J Physiol. 2002;545:595-614. 
26. Proske U, Wise AK, Gregory JE. The role of muscle receptors in the detection of movements. Progres Neurobiol. 2000;60:85-96.

27. Herrington L. Knee-Joint Position Sense: The relationship between open and closed kinetic chain tests. J Sport Rehabil. 2005;14:356-362.
28. Miniello S, Dover G, Powers M, Tillman M, Wikstrom E. Lower leg cold immersion does not impair dynamic stability in healthy women. J Sport Rehabil. 2005;14:234-247. 